\title{
Schematization of Enterprise Architecture Models for Ontology Validation
}

\author{
Joe Essien $^{1}$ and Samia Ousenna ${ }^{2}$ \\ ${ }^{1,2}$ School of Computing and Technology, \\ University of West London, St Mary's Road, Ealing \\ London
}

\begin{abstract}
In order to maintain agility and flexibility within competitive markets, organizations have been exploring ways of adjusting proactively to innovations, changes and complex events by use of EA concepts as a means to model their business processes and strategies. Consequently, the need to ensure appropriate validation of EA taxonomies has been considered as an essential part of these processes as the requirement ensures that business motivation relates to information systems and technological infrastructure. In this research, an approach referred to as Model Driven Validation Approach (MDVA) is introduced. MDVA allows modeling of EA with validation attributes, formalization of the validation concepts and transformation of model artifacts to ontologies. The transformation simplifies querying based on motivation and constraints. The major contributions of this work are the extension of a metamodel of Business Layer of an EAF with Validation Element and the development of EAF model to ontology transformation Approach. With this innovation, domain-driven design and object-oriented analysis concepts are applied to achieve the EAF model's validation using ontology querying methodology. Two case studies were deployed in the research using the Design Science Research approach. The produced artifacts were evaluated based on the criteria specified in the requirements of the design context with emphasis on functionality, completeness, consistency, accuracy, performance, reliability and usability.
\end{abstract}

Keywords: Ontology, Enterprise Architecture, Metamodel, Resource Description Framework Schema, ArchiMate, Business Strategy, Validation, Motivation.

\section{INTRODUCTION}

The major objective of Enterprise Architecture is to provide architectural principles, frameworks, methodologies, processes, tools, knowledge base and techniques that can support the mission of the enterprise. But the multi-dimensional interests, nonstandardization of definitions and principles of EA has led to adoption of heterogeneous approaches and modelling techniques in many organisations today [1]. Literal analysis of these EA patterns show many disparate taxonomies, understood by each stakeholder from a different perspective. Though the design and implementation of EA models currently specify predominantly perspectives and alignment of goals, issues regarding validation and harmonization criteria are ambiguous or presented in a rudimentary way. The postulations do not consider the behavioural attributes of the components that comprise a model as a concept that should be subjected to validation. Many authors believe that EA models are not reusable and are designed to actualize a specific goal after which it is archived or at best used as a reference source [2]. Thus model validation has not been properly defined and most definitions tend to specify the expected results of implementing an EA against predefined goals or established levels of maturity. However, this conception has been contested as incomplete and unsatisfactory as affirmed in many past and recent surveys [3], [4]. As modelling of enterprise continues to influence the way many organisations represent their business strategies and technologies, there is a commensurate growth in knowledge base with resolute lessons gained. However, the development of criteria and methods for evaluating architectures have been given less importance when compared with the development of various architectures and modelling methodologies [5]. Without an extensible and comprehensive validation method, it is difficult for enterprise to evaluate the usefulness of architectures as complex architectures are intricate and difficult to understand by stakeholders.

In recognition of this need, the use of maturity matrices to benchmark as-is against to-be scenarios has been prevalent in many cases [6], [7], [8] as a means to validate EA. While this has been deemed sufficient in certain situations, it has also been argued that it is inadequate as it is subjective and not based on constraints that constitute the model artefacts [9], [10]. 
In the proposed method we used ontology for the validation of EA artifacts. While the use of ontology is not entirely new, the concepts especially as it relates to schematization and resource description frameworks present a fortuity which can allow the development of a different approach for validation of EA models [11]. Thus this research presents EA model validation from a perspective different from the commonly used maturity matrices, balanced scorecard and reference models and espouses the use of interrogative constructs on ontology derived from model artefacts to confirm that the EA model meets the intrinsic goals defined by their motivation.

This paper is structured as follows; the second section describes problems associated with validation of EA; section three presents the contributions of this research; section four explicates the theoretical background for EAF, Motivation, Modelling and Ontologies. This section also delves into the conceptual coherence of modelling, models and ontologies and expounds on the elements that constitute the validation artefacts. Section five presents the theoretical concepts that define the research to ground the research and applies the approach to extend ArchiMate Business Layer Metamodel with Validation Elements. This paves way for the conceptualization of the MDVA with a workflow diagram to illustrate the activities involved. The section is concluded with the introduction of the experimental studies used in this work. Section six presents the formalization of metamodel to enable ontology transformation and develops the Reference Description Framework Schema, Queries and validation artifacts. Section seven presents the transformation of the metamodel to ontology and the associated metaphor. The work is evaluated in section eight. The section correlates the queries applied to the ontology, formulates and applies a strategic framework for the research evaluation and states the limitations of the approach. Section nine concludes the research and states areas of further research.

\section{PROBLEMS WITH ENTERPRISE ARCHITECTURE VALIDATION}

In many implementations of EA, validation has been confined to the use of maturity matrices, balanced scorecards and reference models. Though these approaches have been explored extensively, collaborative works have indicated that the approaches do not provide the sustainable capability needed to validate EA Framework (EAF), models or provide the descriptive semantics for the components with respect to their role in actualizing business behaviour, motivational goals or traceability [12]. A common weakness to these approaches for EAF validation is that the models created are not usually at the same level of abstraction. With the lower levels of abstraction being more detailed than the higher levels of abstraction, there are either disjunctions or multiple conjunctions between the artefacts. The variation in levels of metaphors and semantics creates inconsistencies between the taxonomies and layers in EA models thus making it difficult to assign commensurate weighs of measures, specifically for methods such as levels of maturities.

The broadness in terms of scope, proliferation of concepts, use of heterogeneous and sporadic modelling approaches have been suggested as the basis for the complex and spasmodic challenges that exist today in EA validation.[13,14]. In an effort to streamline these complexities, many EA practitioners primarily recognise four facets of EA, described as business, application, information, and technology perspectives Fischer et al., [1]. However, the determination of what should constitute their categorization has been discursive. Often homogeneous artefacts deploy varied or sometimes indistinct information components to conceptualise similar paradigms for process management and change [3]. For instance, many EA architects have augmented these perspectives to include strategy, organization, culture, data, integration, security, infrastructure, solutions and more [15]. Still it is not uncommon to observe other elaborations created by fusions of these categories so that more convoluted perspectives of the abstractions are aggregated as architectures.

Without the harmonization of EA concepts but with continuous systematic omissions and inclusions of components as elements of EAF, attempts to integrate with various existing technologies and adaptation to multi-facet domains, an immense challenge confounding enterprise architects is that none of the frameworks can be asserted as generic and capable of satisfying most aspects of the enterprise identified collectively. Very significant in this aggregation is that there is no categorization of any EA perspective that reflects on need for validation of EA model, evaluation or testing as a concept that should be encapsulated within the EAF. EA methodologies that predicate verifiable patterns for business behaviour are either lean or docile in specifying an approach for validating the model artefacts against motivation. While many practitioners contemplate on the benefits of validation, most EAF modellers are not clear on how this can be achieved nor assert definitively that it is unnecessary [16]. Be that as it may, almost all enterprise architects know that at some point in their EA project endeavour, their prototypes would have to be proven or justified especially when subjected to broadened contexts, challenges of change management, risk assessments, integration, business dynamism and regulatory compliance. This is because complexities in EA taxonomy have serious consequences which are closely related to the numerous failures in IT projects as affirmed in many past and recent surveys [17],[18].

In view of orthodox postulations that EA can offer the insight needed to balance requirements for facilitating synergy from corporate strategy to recurrent operations, EA models continue to be developed using divergent Enterprise Architecture Modelling 
Language (EAML). This puts high cognitive strains on modellers as the correlation and interpretation of artefacts and notations often become difficult to understand especially to the non-technical users of the system. Therefore many models produced in many organizations make meaning only within those organizations. The implication of this is that as the importance of the adoption of EA continues to grow, these already identified complexities are amplified as they are not often reviewed with definitive progression with the advancements in modelling tools. Consequently, these anomalies make it impossible to develop or adopt a generic approach for validation of many artefacts in the framework.

Many EAML adopt their own unique notations, symbols and protocols. This impairs interrelation accross the various domains. The dependence on domain specific modelling notations implies that across the various enterprise models, there exist inconsistencies, gaps and overlaps that make validation superfluous in some instances and unreliable in other scenarios. Therefore a need for amalgamation of heterogenous metamodels through a common unified ontology is often considered hypothetical for addressing these concerns.

Considering the significance of models within enterprise architectures, it is incontrovertible that the requirement to encapsulate validation into all aspects of EA modelling would be a compelling necessity. Evidence suggests that with time the requirement to present the effects of change on integrated views of the enterprise along all phases of modelling would be mandatory [2]. For instance, in recognition of this exigency many regulatory bodies have of recent heightened awareness of model risk with expectations that enterprises identify key models that need periodic validation in order to confirm their accuracy. This will not only ensure consistent alignment between business functions and information systems, it will also bring a common understanding between business stakeholders, developers and the users. Accordingly, this suggests that the existing validation approaches can still be improved upon perhaps by the adoption of a methodology that can transform a model to a common platform, viewable and testable through unified schemas as attainable with resource description framework. Fundamental to this postulation is the inference that since most EAFs can be transformed to ontology; the means for validating ontology schemas provided by their service resources can be extended and adapted to validate the EA models even if they are created with heterogeneous Enterprise Architecture Modelling Language.

Based on theoretical principles for change management [19], [20], enterprise systems and goal evaluation, this research advances a novel multi-conceptual perspective for addressing challenges of EA validation. The methodology which is original and unique produces as outcome artefacts that represent assessment archetypes for metamodel, models and traceability. The proposal extends the traditional perception of EA validation beyond the level of balance scorecards, maturity matrices and reference models to proffer an advanced metaphoric approach that generates new understanding of the levels of validation in relation with motivation. It argues that modelling of EAF with motivational attributes as opposed to the traditional embellishment of structural classes and relationships is critical for ensuring the actualization of business goals and intrinsic values of the enterprise. The methodology articulates in details the workflow for EA validation implementation, the query approach, and the factors that influence the outcome and results. Furthermore, the convergence of illustrative perspectives in this work brings innovation to EA model management, change management and formalization concepts to bear on a subject which traditionally has been of predominant concern to EA practitioners.

With regard to the challenges associated with EA, this work is limited in its scope in order to focus emphasis on the foundational aspects of modelling that relate specifically to validation. Though models created with heterogeneous semantics can be applied with the methodology presented in this work, focus is placed on models created for the business layer of the EAF. The rationale for this confinement is because the business layer primarily defines the requirements of the other multifarious levels of the EA taxonomy. Thus the effective validation of this base abstract implies that pragmatic means for validation can be ingested into the entire framework. It would also allow the case scenarios to be modelled based on specific viewpoints of business concerns, constraints and goals defined from motivation. The requirements for the modelling of the business layer are considered integral to the framework entities and cascades though the multiple levels of abstraction. Accordingly, these reasons form the justification for this research and provide the grounds for derivation of the research contributions.

\section{RESEARCH CONTRIBUTIONS AND BASIS}

The aim of this research is to extend an EA Modelling Language by introducing capabilities that allow validation to be performed on instantiated models based on motivation from divergent perspectives. To achieve this aim, it is intended that the models would be formalised based on definite views and perspective. Though many presumptions have been put forward to argue that formalization permits the systematization, refinement, and methodological clarification of models [21], the association between these presumptions and various assertions are still subject for speculations. What is however common amongst these presumptions is that formalization makes possible the identification and formulation of unresolved problems [22]. Thus leveraging on the grounds of these presumptions, there is no doubt that formalising and enhancing model with validation extensions would add 
clarity in the presentation of its taxonomy, terms and goals that need to be achieved. It will also establish traceability and more transparency in visualising the effect of change thus exposing gaps and overlaps.

Therefore the research aims to adopt an idealization concept to ascertain the values of the EAF proposition. A derivative axiom that represents the outcome of the transformed model would be factorized to create resource description graphs and schemas that can be validated using natural query language semantics. To execute the query semantics, validation metrics are proposed and used with the validation element extended from an open and independent enterprise architecture modelling language. The metrics which in itself spans most of the specification for model validation as defined by Control Objectives for Information and related Technology consists of five validity criteria to support goals realization, model traceability, motivation assessment, business behaviour analysis and perspective visualization [23]. These validation metrics are applied at the business layer of EA on aspects that encompass critical qualities of model validation. One major advantage of this approach is that it compliments constraints validation specified by motivation. Additionally, a consistent and systematic validation approach can express more specifically constraints using triples thus play an important role in validating the model more efficiently to ensure reliability and traceability.

\section{FUNDAMENTALS OF EAF, MOTIVATION ARTEFACTS, MODELLING AND ONTOLOGIES}

Motivation model is an EA conception that identifies aspects that aid the actualization of business strategy through graphical representation and relationship between the factors of the business plans and intentions. At the centre of motivation model are schemas and structures for developing, communicating, and managing business plans in an organized manner. The Business Rules Group states specifically that the Business Motivation Model should perform identify factors that motivate the determination of business plans; identify and define the elements of business plans and indicate how all these factors and elements correlate [24]. In most implementations, elements of motivation model are developed from a business perspective and stakeholder's viewpoint with the aim to develop business models for the elements of the motivation. In this manner, motivation is the foundation for activities and connects system solutions firmly to their artefacts.

Notably, amongst many existing EAML, the ArchiMate Modelling Language [25] demonstrates this concept of motivation modelling very distinctly. In ArchiMate, Motivational models are used to explain the reasons that underlie the design or change in the EA. It also influences, guides, and constrains the design of the model by use of artefacts that represent goals, principles and requirements [26]. Thus it has been suggested that Motivation model is a blueprint design that can support a range of EA methodological approaches [27]. Implementation of the Motivation model results in a set of concepts that act as a checklist of factors to be considered in the architecture, a standard vocabulary and a flexible model that supports artefact development processes.

There is no doubt therefore that the impact of motivation on enterprise modelling brings the business rationale within business scope; focusing on (a) specific deliverables (goals and objectives), (b) how it intends to achieve them (its strategies and tactics), (c) what will govern the approach (business policies) and (d) its business behaviour (assessments of the impacts of drivers). It also provides a high-level structure that supports fundamental associations with three aspects of EAF and business models namely Business Processes, Business Rules and Organization Roles. In many literatures and EAF implementations such as the Zachman Framework [28] and TOGAF, there exist sophisticated declarative mechanism for specifying goals and other model's motivations [29]. These specifications are related to business concern and standardised high-level logic for business behaviour.

\subsection{Significance of Modelling, Models with Ontologies}

Often, business concerns require visualization of collection of information resources, processes and goals in an articulate and structured configuration. The use of metamodels, models and ontologies provide the means for expressing the attributes and relationship between design artefacts, and establishes the rules that guide their associations. The application of modelling in EA enables clearer visualization of logical representation of business concerns and the interpretation of their distinctive peculiarity from a source metamodel. In corroboration with this backdrop, ontology expresses the semantics of the modeling concepts whose syntax is defined by the metamodel. Ontologies provide the vocabulary of the metamodel's empirical domain and can facilitate its validation. Thus the most fundamental intention of ontology is to describe reality represented by a metamodel as an explicit specification of a conceptualization. Therefore intrinsically, while an ontology is a set of logical axioms designed to provide for the intended meaning of a vocabulary, it formally defines the relations among terms in a metamodel using a set of inference rules. The inference rules are deployed in this study to effect validation of the model as the joint use of metamodeling and ontologies allow for explicit description of knowledge for the domain. 


\subsection{Validation Elements with EAF Artefacts}

To enable testing of the model, validation artefact are identified as test basis and categorized relative to the model components. Specifications from the business behaviour consist of concerns, test scenarios, constraints and expected outcomes. This concept is adopted from Behaviour Driven Development (BDD) approach which principally defines how software development should be managed to achieve business interests as well as ensure technical awareness. At the heart of BDD is a consideration for unit testing and acceptance testing and this has proven very successful as a principle for software development. The attributes of the artefacts in some cases extend to include multiplicity definitions for the object and specifies the types of mutuality of properties and relationship annotation with other artefacts. The test scenarios contain data segregation from constraints and goals in relationship with some measurement metrics. Figure1 illustrates this classification.

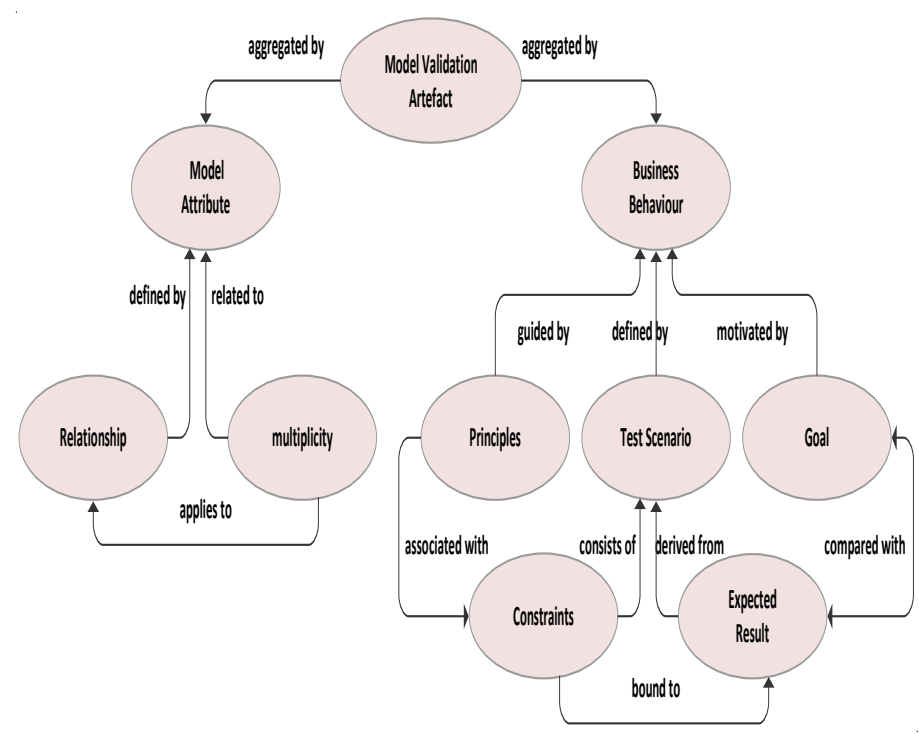

Figure 1: Classification of Model Validation Artefact

Test scenarios specify procedural data used in a confirmatory way to verify that a given set of constraints to a given business behaviour for given goal produces expected result. Test data is produced in a focused or systematic way adopting the VPEC-T concepts. VPEC-T analysis is a thinking framework that comprises of a collection of mental filters. It provides a simplified language for preventing loss in translation from business needs to IT solutions [30]. The purpose of the Model Driven Validation Approach is to validate viewpoints of a model iteratively, across the three aspects (Information, Business and Structure) of the ArchiMate business layer by testing attributes of the model elements against goals in the motivation Extension. The method adopted in the MDVA consists of both the behavioural and the structural attributes of the EA components conforming to the theoretical principles for model validation rules [31].

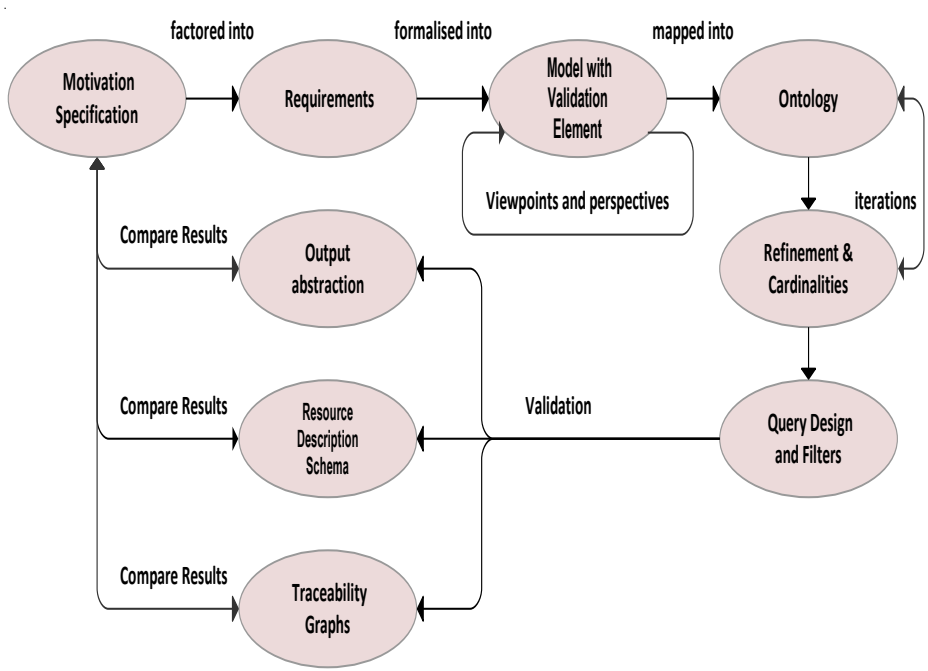

Figure 2: EA Validation Workflow Cycle

The design of the MDVA is conceptualized from the ArchiMate Motivation Extension by deploying motivational constraints on artefacts of the business layer elements of the core ArchiMate. The methodology iterates correlations of motivational elements 
over the taxonomy to establish extent and coverage of the business behaviour defined. Through the validation process, gaps and overlapping functionalities are also identified allowing the model to be more concise and purposeful. Figure 2 shows an overview of the morphology of the MDVA concept proposed in this research. Validation themes are defined by a set of motivational specifications for the components to be tested in the model. The validation element modelled with the ArchiMate Business core defines the metrics and what types of test are to be carried out on the components and the expected results. Test attributes are specified at this level. Constraints expressed within stakeholders concerns and principles are applied on the selected components to construct the test basis. This stage is iterated to develop the various viewpoints such as process and functional models that would be mapped into the ontology. The development of the ontology is also an iterative process that allows for refinement of domains, ranges and cardinalities. By implementation of the BDD concept, language semantics is built with the precondition and post conditions adduced to form the queries. Traceability amongst the other components is established by exerting associated relationships. Evaluating the result yields three outcomes;

- Output abstractions that allow comparison to ascertain if the tested goal is realized,

- Resource Description Framework Schema that provides the construct to executing queries,

- $\quad$ And Resource Description Framework graphs that facilitate traceability.

To enable the identification and rationalization of the validation metrics for the EA models, study of standards specified by bodies such as COBIT, IEEE, ISO and TOG were conducted and cultured with model driven composites. Several validation metrics and elements were analysed in relation to their characterization and congruity. Through heterogeneous sampling method, five of the elements which exhibited properties that reflect enterprise architecture maturities were selected. These elements are redefined in context with this work to give it distinctive meaning, relationship and representation. These are Goal Realization, Perspective Visualisation, Behaviour Analogy, Model Traceability and Constraint Assessment.

\section{EXTENSION OF THE ARCHIMATE BUSINESS LAYER METAMODEL WITH VALIDATION ELEMENTS}

The validation extension presents an extension of a generic business layer of EA with embedded artefacts for validating its usability and important specifications for key performance indicators, business behaviour, perspectives and their relationships. To create a model-driven taxonomy that spans the business and motivation layers for validation, a schema designed to relate the motivation elements with core ArchiMate is presented in Figure 3. It depicts the conceptualization from a stakeholder's perspective and the transformation of concerns with principles and constraints through Assessment to Goal and Requirement.

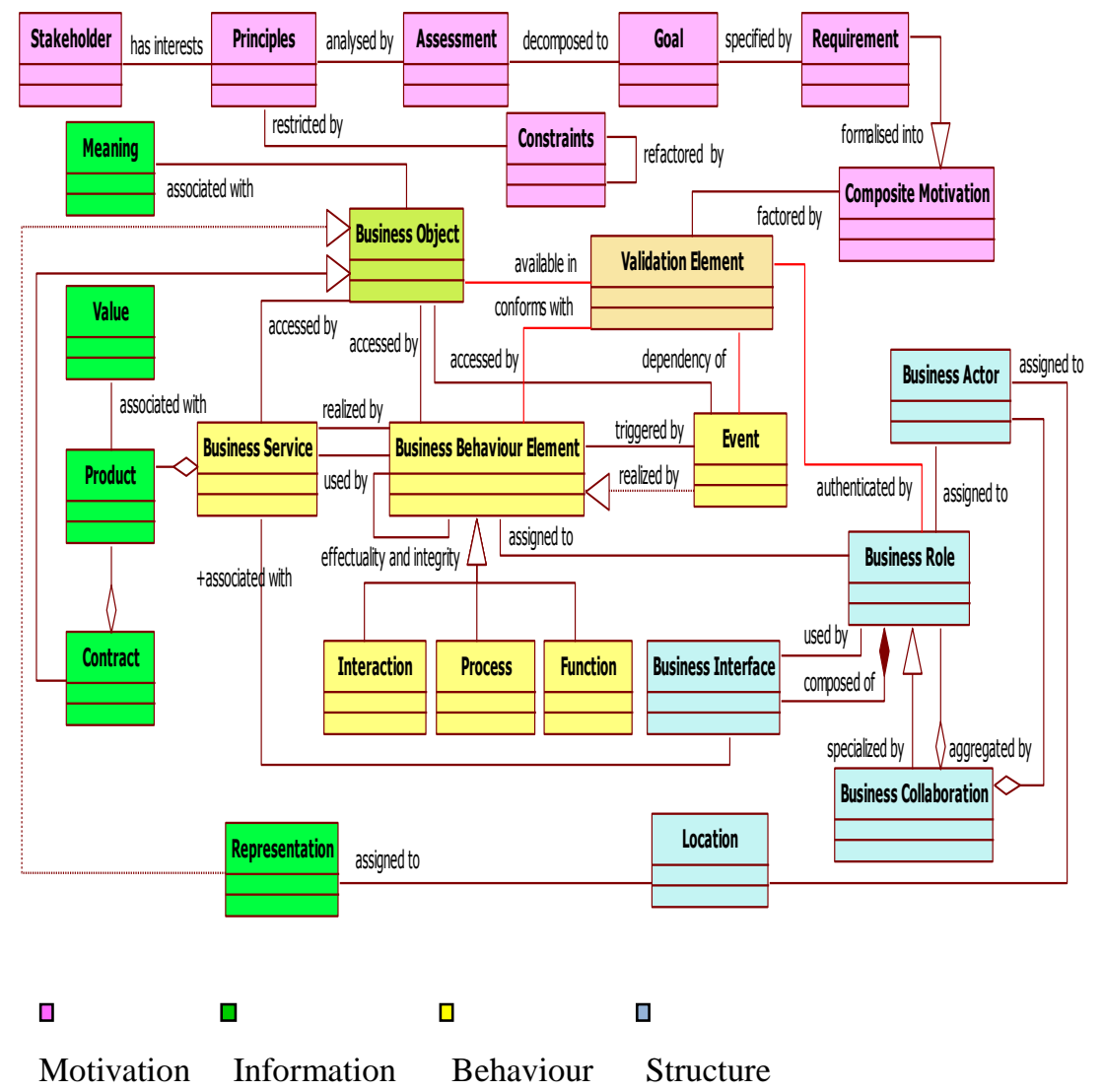

Figure 3: ArchiMate Business Layer MM extension with Validation Elements 
Within the ArchiMate Business Layer, the Validation element is represented as high-level information artefact. Goal on the metamodel extension is associated through Requirement and Composite Motivation to Business Behaviour elements through the ambience provided by Validation Element. This sub classification allows further query relation to be distinctively applied to the business processes and business function to ascertain the artefacts integrity and effectuality respectively. Requirements which specify the Goals defined in Motivation appropriate a theme to be adopted by the evaluation iteration process. The query structure and semantics of the Validation Element allows criteria specified by constraints to be tested for instance against Business Objects, Business Role and Business Event.

Further, the metamodel presented in Figure 3 depicts a high-level conceptual construct that abstracts information, associates business behaviour with organisational structure and aggregated relationships. Business viewpoints are derived by analysing Business Roles which are composed of primarily Interface, Collaboration and Actor. This is deliberate as the intention of this work is to espouse the alignment between the business strategy and motivation.

\subsection{Conceptualising the MDVA}

The Motivation Driven Validation Approach (MDVA) enables the testing of artefacts, relationships, constraints and guides in the actualisation of particular goal in the Motivation. MDVA is realized across the business and motivation layers of ArchiMate. The diagram in Figure 4 depicts the concepts. There are three swim lanes of the approach across the Business Layer and Motivation Extension of EAF, modelling with Validation and Ontology transformation. The first section delves into the value of the proposition and decomposes the EAF to its motivation and components.

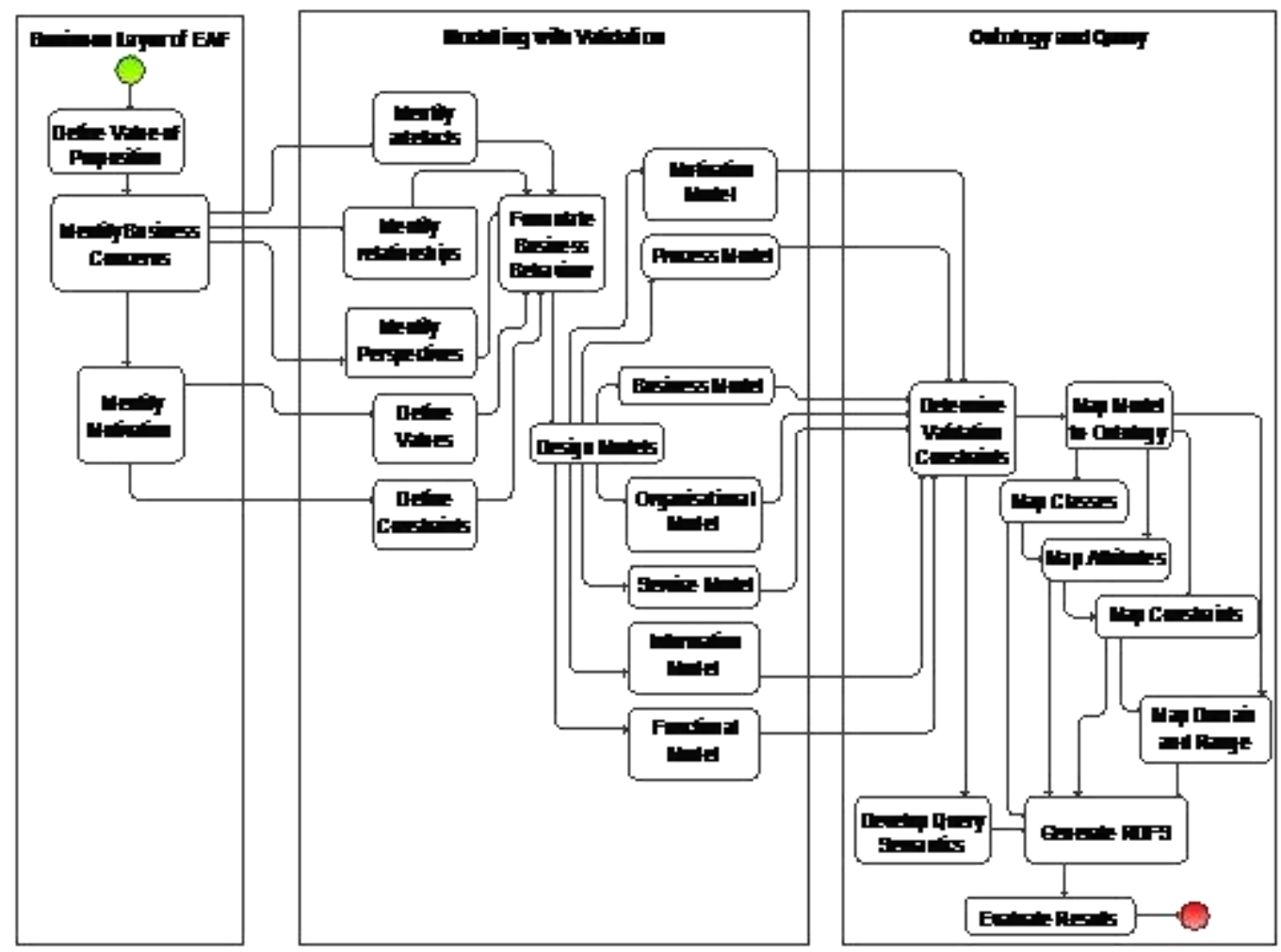

Figure 4: Workflow Diagram for the MDVA

The second section depicts the modelling aspects and comprise of metamodels and its instances. Case studies are applied at this level to exemplify the methodology. It also illustrates the realization of business behaviour from the Business layer and Motivational constraints. The model instance extended with validation concepts are analysed, extrapolated and integrated from specific business behaviour. The third group of activities handle the ontology creation and all aspects that relate to mapping, building of query semantic and generation of the Resource Description Framework (RDF). The model instances created with validation constraints are mapped into its equivalence class, properties, domain and ranges in the ontology framework. This is transformed into RDF Schema (RDFS) and traceability graphs. The resultant triple constructs of the RDFS are then executed with a series of queries to validate the model against motivation goals.

The process of ontology mapping expressed in the third group of activities in this workflow is described and specified as follows; 
i. Given a model, identify the various testable artefacts to ascertain the values of the proposition. VPEC-T can be used here as a thinking framework.

ii. Identify the business behaviour associated with the model and associate goals and constraints.

iii. Identify the various motivational, structural, information and functional models.

iv. Decompose the model to class representation annotating its associated attributes and relationships.

v. Identify testable artefacts for the nodes, domains and ranges.

vi. Identify relationship that exists between the nodes and slots. This is required for the development of traceability.

vii. Identify attributes of the node that relate to constraints. This is required for the development of the features and scenarios of the ubiquitous language.

\subsection{Experimental studies}

For this research, two separate implementations of the Model Driven Validation Approach were considered.

The case studies focused on using the extended validation elements of a modelling language to create models that conform to the metamodel of Business Layer and to exert validation through querying the RDFS. The outcome of the queries issued with the taxonomy of the RDF graphs produced are analysed and then subjected to evaluation adopting the supporting theories of this work. The objective of the case studies were to provide an in-depth application of the methodology, detailed examination of artefacts and an empirical inquiry that allows the investigation of the hypothesis proposed within a real-life context. The two case studies are briefly described.

\subsubsection{Case Study A: University of Middle England Laptop Loan Scheme (UME-LLS)}

The University of Middle England (UME) is worried about its future. After some analysis, the key reason seems to be that students and the resources used for teaching and learning activities are badly aligned and that some of the modules assume that all students have their own laptops (which are not always the case). As a result of this analysis, UME decides to implement a laptop loan scheme whereby students who do not have their own facilities can loan them from UME at no cost. The scheme raises questions about which modules require laptops, whether the current teaching and learning rooms are suitable for laptop usage, and whether UME has suitable software with appropriate licenses for use by students. In order to implement the laptop scheme, UME decides to use EA. Having used EA to design a migration path, UME wants to determine whether the scheme is consistent with its business goals and to understand how its new architecture operates. A working group is tasked by the UME executive to come up with a description of the information structures relating to student teaching and learning.

\subsubsection{Case Study B: University of West London Student Internship Project (UWL-SIP)}

Graduate employers, particularly in competitive fields, increasingly view relevant work experience as a must, with employers seeing internship schemes as a filter to identify new employees. Students see an internship as a valuable life experience that can lead to improved academic achievement and a way of making career contacts. Student employability agenda requires institutions to respond by demonstrating that they have excellent resources and processes in place to support students in securing graduate employment. The UWL sought to extend this provision to enhance student employability and to build better links between the university, businesses and community projects.

An EA modelling is planned to concentrate on the goal, information systems, processes and services modelling. The EA approach should review the graduate internship programme, analyse the school/course internship programme stakeholders' requirements and review of the institution technologies and applications. The major motivation for this study is to build the as-is and to-be architectures with focus on internship provision and also proposes a solution model for the programme using an EA approach. This will include the different viewpoints; i.e. the business processes, the information models, the interaction of the institution with external systems and development of services and portals.

\section{ONTOLOGYTRANSFORMATION}

The theoretical principle for goal evaluation and change [31] plays an important role in grounding the principle provided as the basis for logical and conceptual exposition of principles for mapping model to ontology. The Theory of Change is widely acclaimed to yield dependable result as it is based on a series of critical thinking exercises aimed at providing a comprehensive picture of the early and intermediate variations in a given scenario. However, applying this principle, there are also some assumptions that are made in this work Firstly it is assumed that the mapping of metamodel will not take into considerations overlaps, gaps and the basic relations that supplement their negative counterpart. This is because generally overlaps are indication of redundancies in the metamodel, gaps are omission which may not be subsumed without appropriate rationalization and negative counterparts represent duplex relations which are taken care of while defining the ranges and domains in the ontology. As a variation, a metamodel artefact can also be completely disjoint with other artefacts. In that case the artefact is also presented as disjoint in the ontology. Thus the mapping to ontology strives to maintain the same intentional interpretations as expressed in the metamodel. 
All mappings in this transformation are normative and unidirectional. A mapping is consummated when there is a set of identical assertions that consist of a semantic relation between map-able elements from model to derivative ontology. The mapping is irrelevant without the originating metamodel or model as its purpose in this work is to embed queries that can allow the metamodel to be validated.

\subsection{Developing the Reference Description Framework Schema and Query}

The transformation of EAF metamodel or a model instance to RDFS allows the ontologies to be validated using a Protocol and $\boldsymbol{R D F}$ Query Language (SPARQL). OWL Protégé is used to build the context of coalition operations and to design the collaborative ontology domain. It also enables the capture of artefacts represented in the Business model. Given the objective of collaborative creation and querying of the ontology, it has been advocated that the primary requirements for ontology is to support the methodological process of building an RDFS that can be queried [32]. In particular, this research provides these functionalities and relates to collaborative business behaviour of the EAF. In order to facilitate the querying of ontology, it advocates building of triples and use of enhanced visualization. The result thus is a well-documented guideline for the approach of ontology design based validation and description of the different mapping of the EAF artifacts.

While many EA practitioners argued that there is no formalised way for mapping ontologies, there is a general consensus that whichever methodology is adopted must maintain congruity, consistency and be well defined. To ensure that this standard is achieved and overlap of artefact during mapping is avoided, a top-down class bottom-up slots approach is proposed. This approach starts by identifying the uppermost artefacts on the taxonomy and associates all immediate subcomponent as branches. This ensures that the maximum association is achieved for each branch as it scales down to the lowest artefact on the leaf without any overlaps. This approach decomposes the metamodel into RDFS Structural hierarchy. To build the ontology, a reverse bottomup approach is used to ensure that all nodes are represented with no dead leafs.

\subsection{Content Categorization for Model to Ontology Mapping}

Content categorization is a link-based classification approach used in isolation or in conjunction with text-based classification to assign artefacts to one or more predefined categories based on their contents [33]. A number of modelling classification and knowledge management techniques have been applied to content categorization such as nearest neighbour, Support Vector Machine, Voted Classification and Neural Networks [34]. More recently, some preliminary studies have attempted to apply content categorization techniques into merging and mapping of ontologies. Lacher, [35] presented an approach using supervised classification (Rocchio) for ontology mapping while another method referred to as FCA-MERGE, based on the theory of formal concept was proposed by Stumme \& Maedche [36]. Though these approaches are veteran, their analysis which stipulates that generation and mergence of ontologies should follow a bottom up approach guided by application-specific instances is still widely practiced of recent. In our approach, this theory is enhanced by adoption of top-down perspective for implementation of the mapping process and bottom-up perspective for the mapping to ontology. The metamodel to ontology elements mapping are determined by similarity in characteristics per pair. Only the combinations with similarity attributes are considered as equivalent. In order to establish definitions of similarity and to support development of accurate mapping, a framework for the mapping is defined. This is presented in figure 5 and is adopted as the basis for correspondence assertion for OWL.

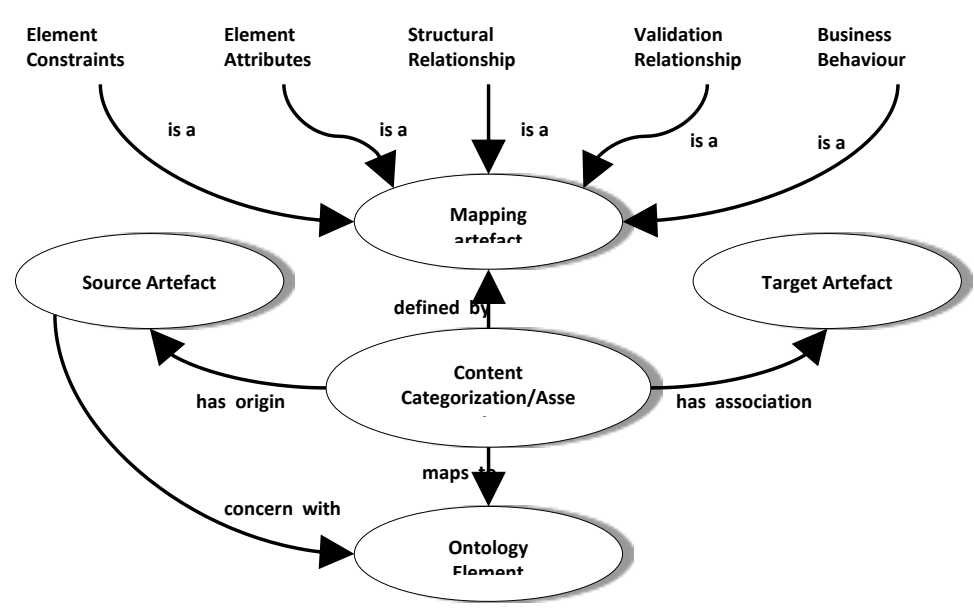

Figure 5: Conceptual framework for OWL Mapping 
The diagram in Figure 5 describes the content categorization as an objectification of the relationship between ontology elements with support for further description of that source and dependent associations. A content categorization is uniquely assigned to the ontology elements. It has also an association in order to provide a way of establishing dependencies and traceability of the artefact within the schema. Definition is also attached to the content categorization in order to establish content and specify how the mappings of the ontology elements are related. The objective of maintaining artefact's derivation is to provide an explanation for the source of its origin.

The screenshots in Figure 6 and Figure 7 depicts this transformation for EA model; from artefacts to classes and relationships to properties. The constraints and validation metrics are defined for domain and range.

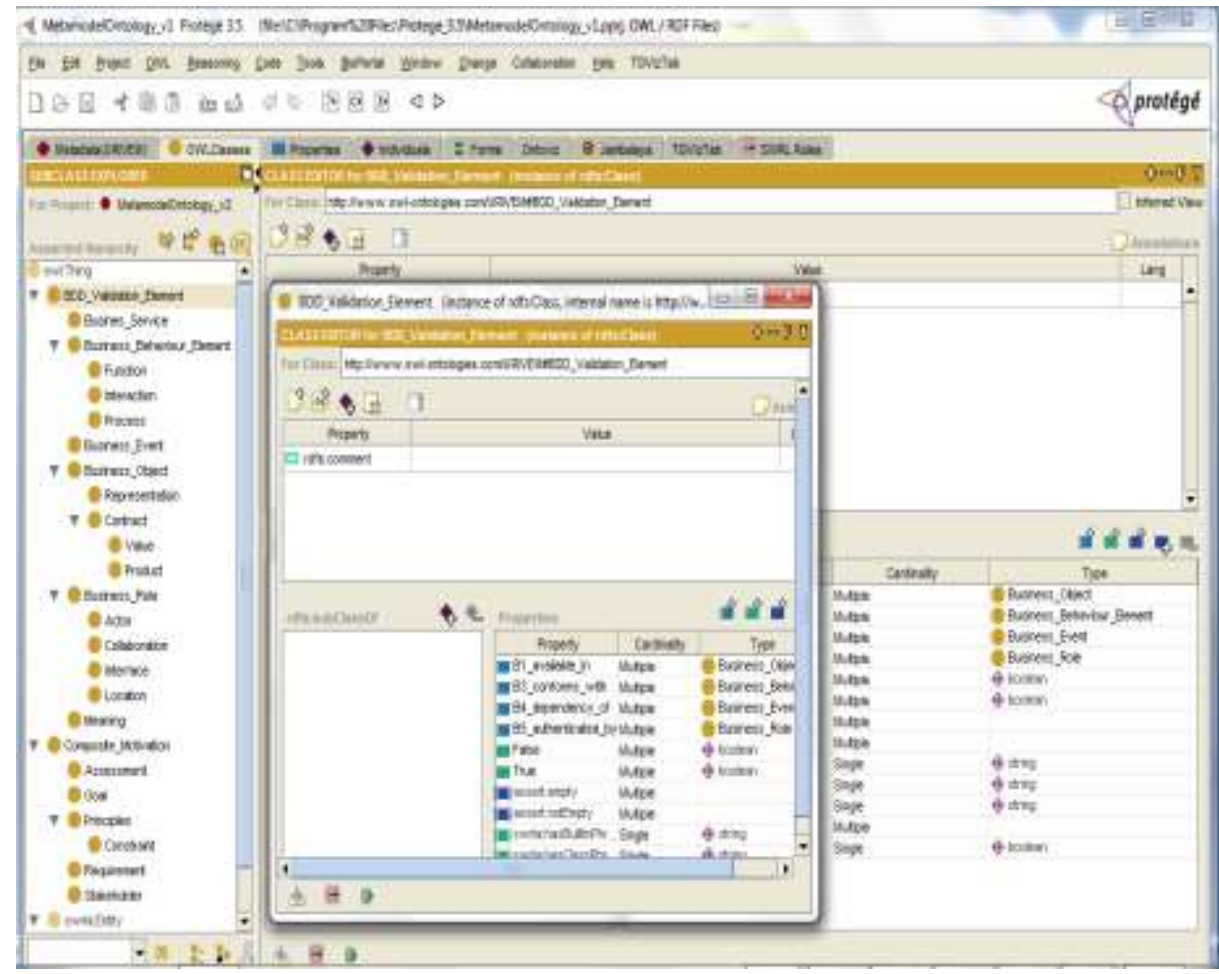

Figure 6: Association of EA artefacts to ontology Classes

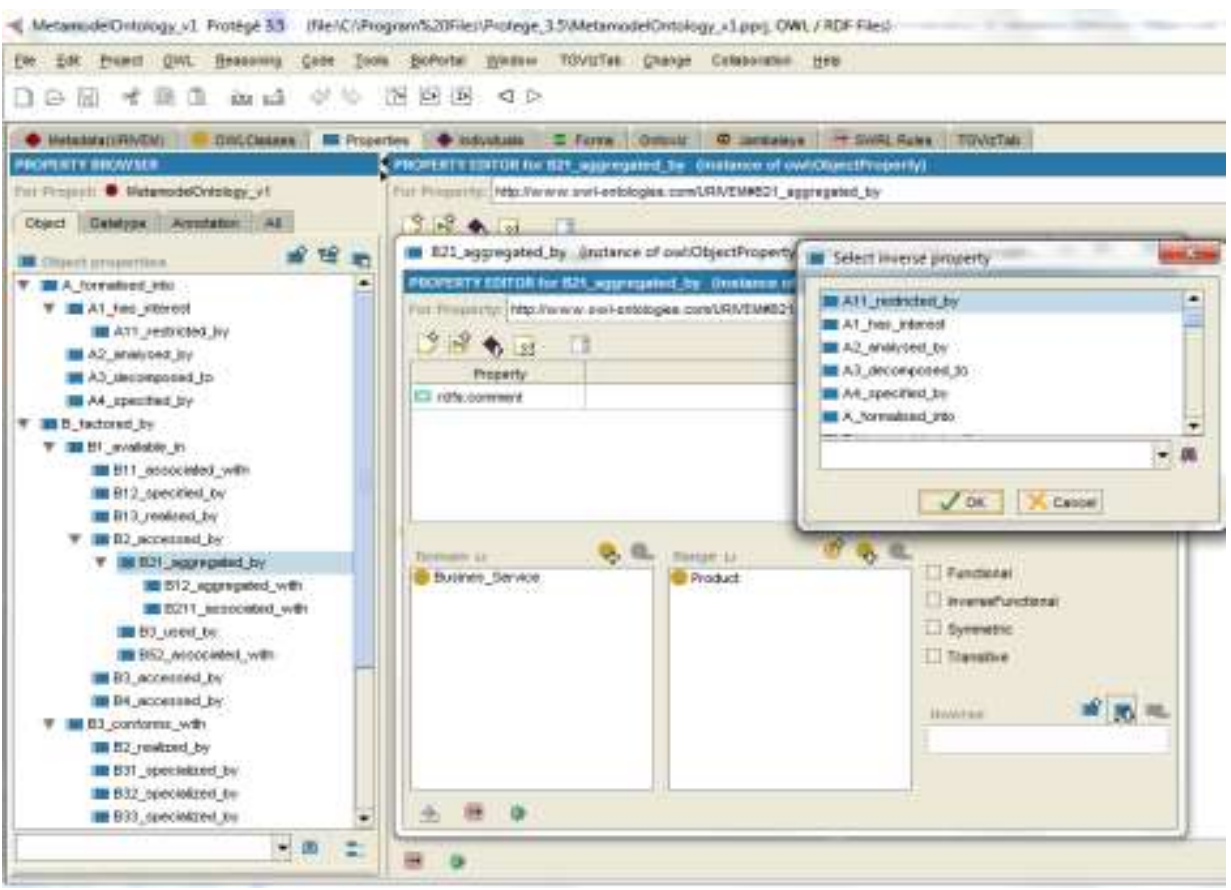

Figure 7: Association of EA Relationships to properties with domains and ranges 
Slots link individuals from the domain to other individuals from the range. Unlike applied in conventional ontology derivation, a range can be used to set constraints that needs to be validated as well as axioms for querying. The transformation also ensures that all individuals which represent the components belong to domains so as to provide a mechanism to relate objects from the ontology to the business artefacts in the EAF. This in turn ensures that both structural and semantic heterogeneity are resolved allowing concerted queries to be performed on the generated RDFS. One major advantage here is that collaborative mappings can be applied to the ontological components. Additionally, with the extended capability to use specialized ranges provided by OWL expressions and the level of granularity is increased.

\section{Correlations and Ontology Queries}

An extensible knowledge representation with all the elements from the EA metamodel is depicted in Figure 8. The vocabulary generated with this ontology forms part of the triplestore that is queried. With this construct, it is easy to build queries by $\mathrm{N}$ Triples with the subject denoting the class; the predicate denoting traits or aspects of the class and expresses a property between the subject and the object.

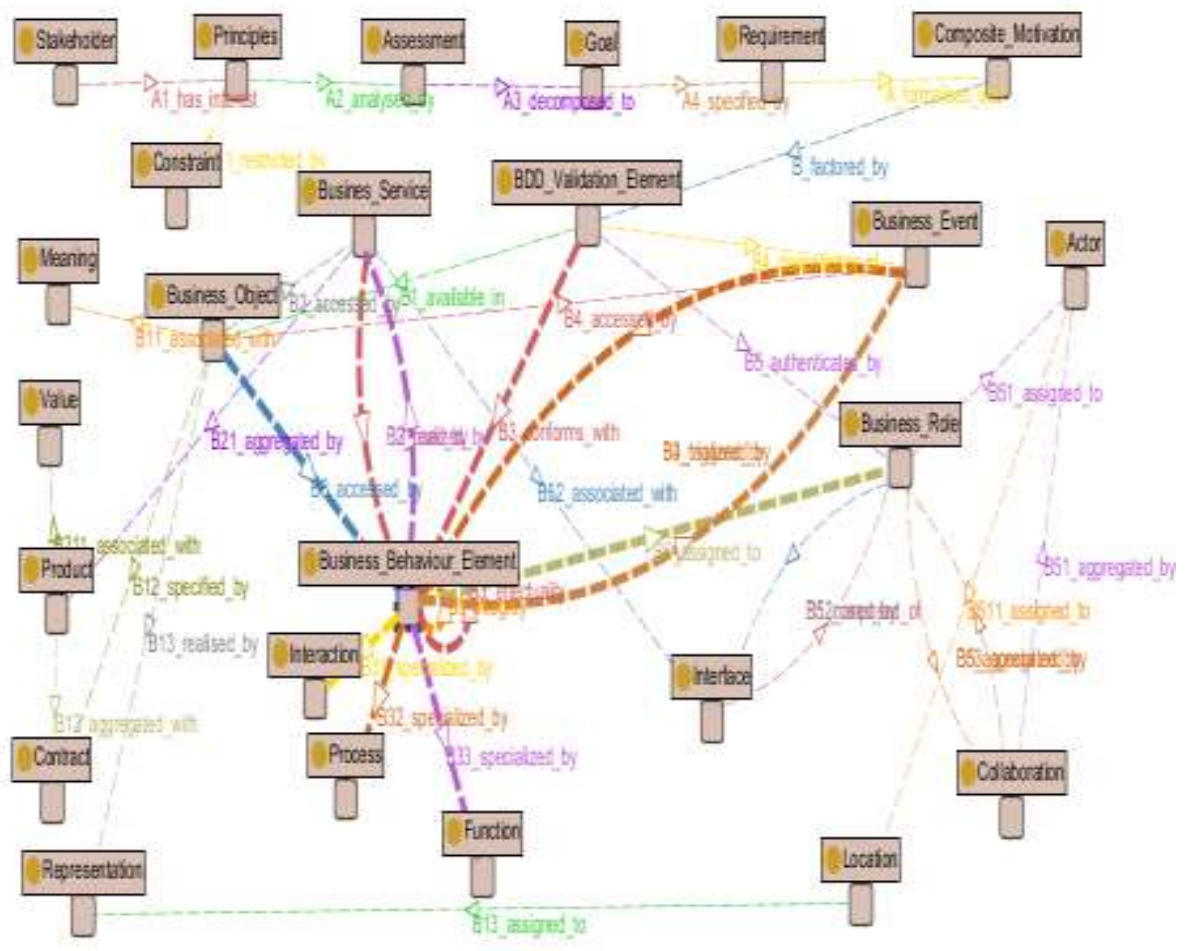

Figure 8: RDFS of the ontology representing the Metamodel

A complete ontology transformation of the metamodel to RDF Graph is depicted in Figure 9. It shows an extensive filtration of traceability used to probe the metamodel exposing the motivation, structure information and business behaviour for selected properties, ontology bindings to domains and ranges that relate to a common viewpoint. 


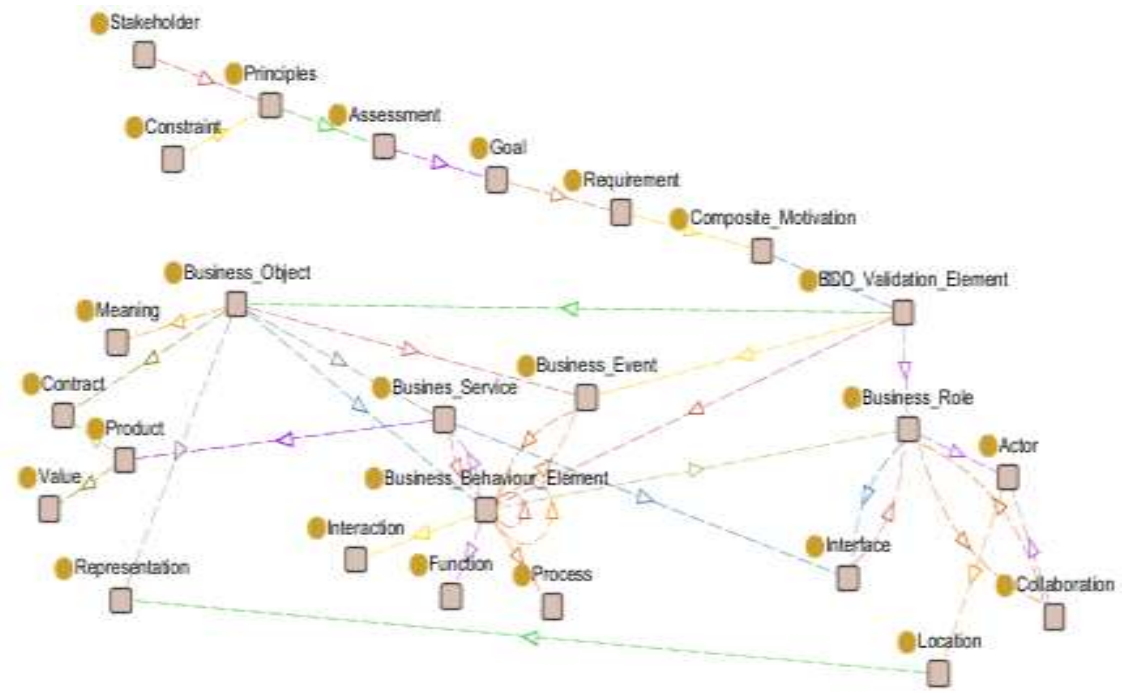

Figure 9: Schematic segregation of RDF into motivation, information, behaviour and structure

\subsection{Querying the Ontology with Reasoners}

While there are several literature on querying ontologies, in this section analytic query operations are carried out to demonstrate whether the transformed metamodel can be validated using the triple stores generated with the RDFS. The OWL uses the inmemory stores approach effectively with its Reasoners to perform abstruse inferences in persistent RDFS stores; which otherwise could have been very difficult to perform. SPARQL is used in many implementations including Knowledge Explorer, Open RDF Sesame and Big Data. While OpenRDF Sesame is a de-facto standard framework for processing RDF data and includes parsers, storage solutions triple stores, reasoning and querying, using SPARQL, it also offers a flexible and easy to use Java API that can be connected to many leading RDF storage solutions.

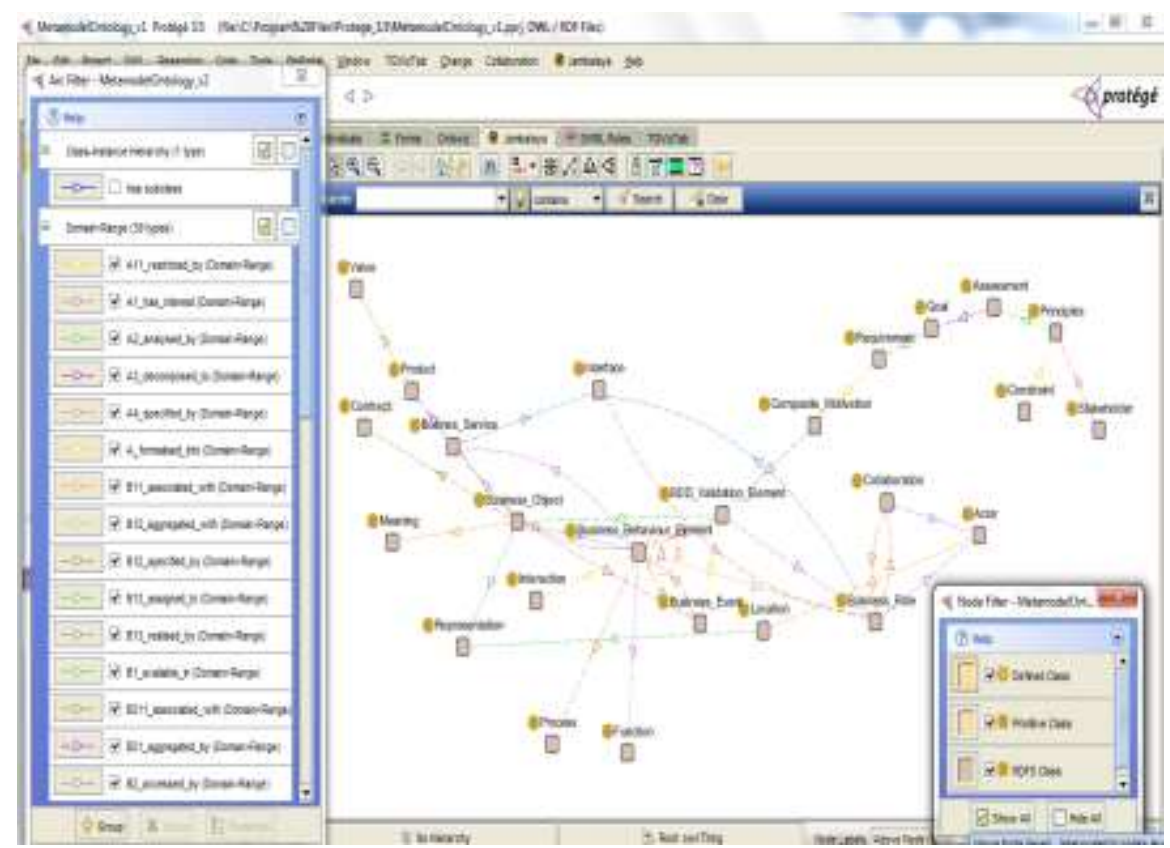

Figure 10: Querying the ontology using the Reasoner

An example of this interchange is shown in the implementation of the extended validation metamodel in Figure 10. The diagram shows a transitive closure on the sub-property and sub-class hierarchies selected on the filter panel.

\subsection{Querying the ontology using SPARQL}

A query language in this context refers to a set of interrogative assertive with similar circumspective characteristics with SQL. Query languages usually fall into one or more of the four categories specified as SELECT, AGGREGATE, UPDATE and DELETE [37]. In this implementation, the SELECT query category which retrieves a persistent state from one or more entities and filters results is adopted. This may be extended to include the AGGREGATE queries which are variations of the SELECT query and groups results to produce summary data. Together the SELECT and AGGREGATE queries constitute the outcome 
reports in this implementation as they primarily focus on generating data for comparison. The UPDATE and DELETE queries which conditionally modify or remove parts or entire sets of entities are not considered in this research as the work aims to verify and not make change in the taxonomy. Querying in this context deploys entities and objects written against domain ontologies instead of rows and columns of database.

Data types for querying the EA instance include the use of literals with the general syntax or an optional data type Internationalized Resource Identifiers (IRI) or prefixed name. The RDF data model itself represents the EA artefacts as graphs consisting of triples with subject, predicate and object and allows SPARQL to make queries that involve artefacts from more than one graph. SPARQL query is executed against the RDF dataset which represents a collection of graphs. A SPARQL query matches different parts of the EA model pattern against different graphs. Thus the RDF graphs and schema are generated as primary test basis for the tests carried out to validate the hypothesis of this research.

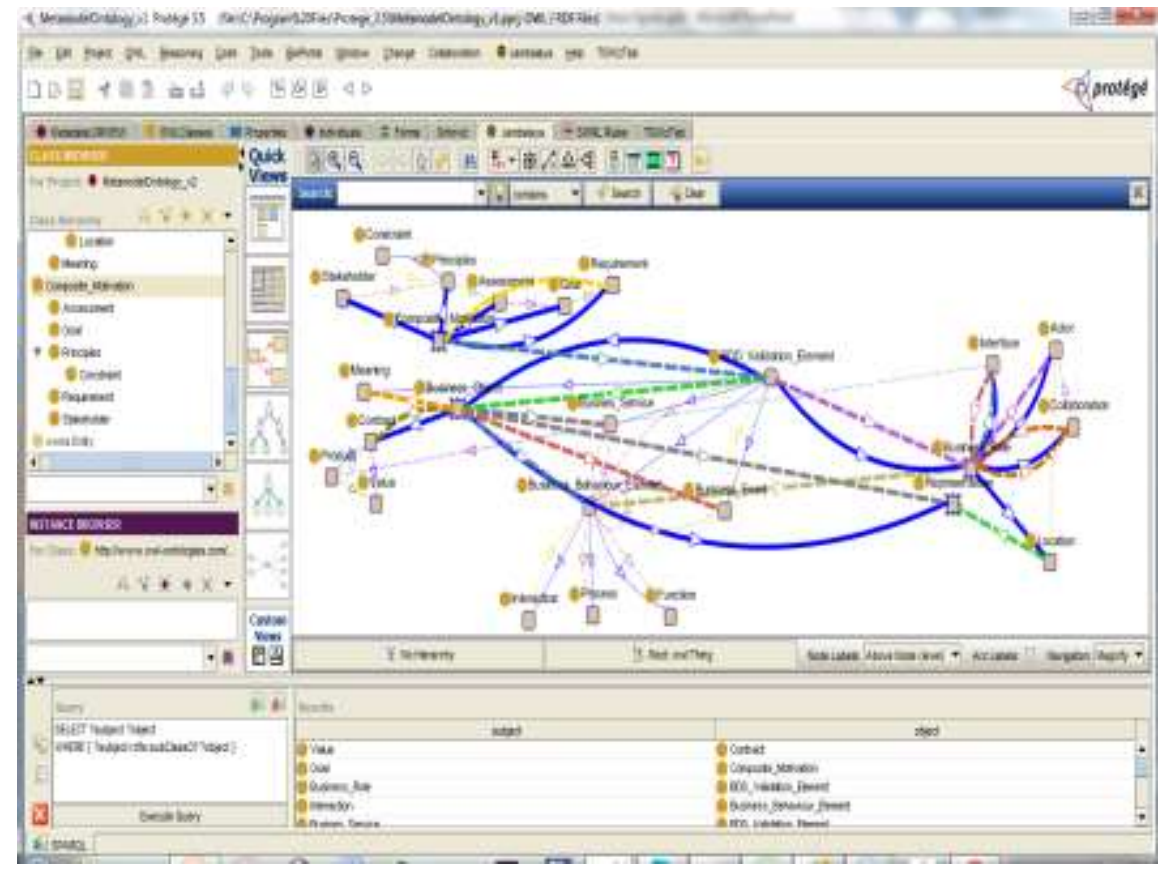

Figure 11: Traceability of class and relationships on ontology using SPARQL

The construct of the SPARQL query is concentric at the triple level applied on the RDFS. The outcome is extrapolated by filtering individuals and classes with specific characteristics or properties amongst many other attributes. An example of this outcome is presented in Figure 11 as a result set and a RDF graph. This example illustrates a direct principle of extraction with respect to the metamodel under consideration but a more extensive validation can be provided in case studies where constraint checking is used to validate motivation in conjunction with OWL assertions added as annotation properties to the selected classes.

\section{RESEARCH EVALUATION}

Evaluation provides evidence that a new technology developed in a Design Science Research (DSR) process achieves the purpose for which it is designed. Without evaluation, outcomes of DSR are unsubstantiated assertions that the designed artefacts if deployed in practice will achieve its purpose. According to Gregor \& Hevner, [38], these artefacts should be evaluated with criteria based on the requirements of the context in which the artefact is implemented. The outputs framed as DSR artefacts usually consist of constructs, models and methods.

\subsection{Formulating Strategic Framework for Research Evaluation}

The purpose of this section is to formulate a strategic framework for evaluation of this work within the context of DSR. A strategic framework serves three purposes. It is used to help build strategies for evaluation of research outcomes, to achieve improved rigor in DSR and to descriptively improve understanding of unstated evaluation implications in the case under consideration. Based on several valuable principles of ISDT [39], this evaluation draws on the principle which distinguishes between ex ante and ex post evaluations. Ex ante evaluation take place before a system is constructed while ex post evaluation take place after the system is constructed. Drawing on this principle, the DSR outputs consist of the metamodel extension, metamodel instances, proposed validation methodology, validation metrics and generated RDFS and traceability graphs. By extending the principles as described by Venable [40], a strategic framework can be formulated by choosing the prominent 
alternatives described above featuring when evaluation takes place, what is actually evaluated, and how it is evaluated. "When" to evaluate may be selected from ex ante, ex post, or both. It incorporates aspects such as the evaluation context (real stakeholder, organizations, views and viewpoints). "What" is evaluated involves choosing between the design processes or the design artefact. This also includes the granularity of evaluation. "How" to evaluate may be selected from naturalistic or artificial forms of evaluation. The strategic framework is designed to be used both normatively to guide the design of DSR evaluation and descriptively to ascertain conformity with extant DSR evaluation principles. The application of the framework for these two purposes is discussed in the following section.

\subsection{Applying the Strategic Framework for Research Evaluation}

In applying the framework descriptively, three main questions are asked; (1) what is actually being evaluated? In the case of this study, EA artefact and its methodology; "what" may also include the evaluation granularity? In the case of this work, evaluation granularity levels are (a) whether the individual artefact was retrieved, (a) whether the business function which involved the artefact was completed, and (c) whether the completed task had a valuable impact on the associated goal or motivation. These granularity levels represent levels of means to achieving the goals and may be graded from level (a) to (c) ranging from low, intermediate and high. (2) How is it evaluated? As applicable to this study, using the naturalistic evaluation approach and (3) when is the evaluation carried out. This can be ex ante evaluation, ex post evaluation or both. To illustrate this evaluation approach, the following assertions are considered based on the theoretical principles for model validation which is grounded with the research to evaluate the approach based on;

i. Principles for Active Validation Level which anchors on the model's quality of functionality.

ii. Principles for Passive Validation Level with underpins traceability and integration and;

iii. Evaluation of the MDVA workflow which describes the steps of the methodology.

The application of scientific theoretical principles presented in this work towards the conceptualization of MDVA is a contribution that provides a solution to issues concerning EA validation and a methodology for objective validation of models. This approach is justified as it provides a clear understanding of several challenges in validation of EA models.

\subsection{Limitations of the Approach}

Some distinctiveness of the Design Science Research (DSR) adopted in this research also constitutes its limitations in some ways. Of these diversifications, few are most prominent. Firstly, DSR emphasizes the domain in which the design activity will take place, thus placing a premium on its innovativeness within a specific context. This contrasts with most other research methods which emphasize increased understanding of design methods often independent of the domain. Secondly, DSR has a closer affinity to disciplines such as computer science, software engineering, and organization science rather than with other cognitive science and professional fields. This implies that internal design cycles are at the centre of design science research projects. Thirdly, DSR is motivated by the desire to improve the environment by the introduction of new and unique artifacts and the processes for building these artifacts. The applicable domain must consist of people, enterprise and technical systems that need to interact and work towards predefined goals. Thus a limitation is that the iterations of DSR would need to initiate a procedural context that must not only provide the requirements for the research but also define acceptance criteria for the final evaluation of the research outcome. Finally, Design science draws from a vast knowledge base of scientific theories and methods that provide the foundations by which the research is grounded. Thus all models created have limitations and are subject to the validity of their underlying theory and assumptions.

\section{CONCLUSION}

As validation of EAF is an area that currently draws very little diligence amongst practitioners, this research presents a novelty methodology through which much research can be initiated. This include amongst many others a case for integration of divergent EAFs through a common vocabulary using ontology so as to allow better congruency, traceability, validation and alignment of business objectives to Information Technology.

Overall, research has indicated that architecture concepts are not sufficiently exploited. One of the reasons is the lack of appropriate architecture representation formalism supporting the characterization of features and properties of enterprise systems at a high abstraction level. Existing enterprise architecture proposals are represented in different ways with neither a rigorous syntax nor semantics. Existing architecture principles are seldom developed to a satisfactory level which allows amalgamation of significant improvement to enterprise architecting.

Areas of further research may include the exploitation of the ability to merge several ontologies developed within the same enterprise, possibly using divergent or cascaded EA framework for effective interoperability and validation. From this work, it 
seems feasible that divergent EAFs can be amalgamated through a common vocabulary using ontology. Further research needs to be carried out to define the parameters through which this can be achieved. There is also an important need to develop an agreed architecture representation language and evaluation method/metrics so that architecture proposals can be properly described, assessed and compared.

\section{REFERENCES}

1. Fischer, Christian, Robert Winter, and Stephan Aier. "What is an enterprise architecture principle?." Computer and Information Science 2010. Springer, Berlin, Heidelberg, 2010. 193-205.

2. Chen, David, Guy Doumeingts, and François Vernadat. "Architectures for enterprise integration and interoperability: Past, present and future." Computers in industry 59.7 (2008): 647-659.

3. Jørgensen, Hans Henrik, Lawrence Owen, and Andreas Neus. "Making change work." IBM Corporation (2008).

4. Bloch, Michael, Sven Blumberg, and Jürgen Laartz. "Delivering large-scale IT projects on time, on budget, and on value." Harvard Business Review (2012): 2-7.

5. Khoury, Gerald R. A unified approach to enterprise architecture modelling. Diss. 2007.

6. GARTNER, Available at: http://www.gartner.com. Accessed: April, 2018.

7. Weston, J., and J. Defee. "Performance Based Enterprise Architecture Planning-A white Paper, 2004." (2013).

8. Lakhrouit, Jihane, Karim Baïna, and Khalid Benali. "Model and application architecture indicators of evaluation the enterprise architecture." New Perspectives in Information Systems and Technologies, Volume 2. Springer, Cham, 2014. 63-71.

9. Beznosov, Konstantin. "Information enterprise architectures: problems and perspectives." Written for the Advanced Topics in Software Engineering seminar given by Dr. Michael Evangelist at the School of Computer Science, Florida International University. 2000.

10. Pereira, Carla Marques, and Pedro Sousa. "Enterprise architecture: business and IT alignment." Proceedings of the 2005 ACM symposium on Applied computing. ACM, 2005.

11. McGuinness, Deborah L. "Owl web ontology language overview w3c recommendation 10 february 2004." http://www. w3. org/TR/2004/REC-owl-features-20040210/(2007).

12. Klein, John, and Michael Gagliardi. A workshop on analysis and evaluation of enterprise architectures. No. CMU/SEI2010-TN-023. CARNEGIE-MELLON UNIV PITTSBURGH PA SOFTWARE ENGINEERING INST, 2010.

13. Sessions, Roger. "A comparison of the top four enterprise-architecture methodologies." Houston: ObjectWatch Inc(2007).

14. Proper, Henderik, and Marc M. Lankhorst. "Enterprise architecture-towards essential sensemaking." Enterprise Modelling and Information Systems Architectures (EMISAJ)9.1 (2014): 5-21.

15. Schekkerman, Jaap. How to survive in the jungle of enterprise architecture frameworks: Creating or choosing an enterprise architecture framework. Trafford Publishing, 2004.

16. Engelsman, Wilco, et al. "Extending enterprise architecture modelling with business goals and requirements." Enterprise Information Systems 5.1 (2011): 9-36.

17. GENECA RESEARCH REPORT ,"Doomed From the Start? Why a Majority of Business and IT Teams Anticipate Their Software Development Projects Will Fail” Available at: http://www.geneca.com. Winter 2010/2011 Industry Survey. Accessed December, 2018.

18. Logica Management Consulting. Failing business process change projects substantially impact financial performance of UK business, Available at: http://www.cgi.com/en. Accessed: January 2014. Accessed December, 2018.

19. Siponen, Mikko. "Six design theories for IS security policies and guidelines." Journal of the Association for Information systems 7.1 (2006): 19.

20. Markus, M. Lynne, Ann Majchrzak, and Les Gasser. "A design theory for systems that support emergent knowledge processes." MIS quarterly (2002): 179-212.

21. Zimmerman, John, Erik Stolterman, and Jodi Forlizzi. "An analysis and critique of Research through Design: towards a formalization of a research approach." proceedings of the 8th ACM conference on designing interactive systems. ACM, 2010.

22. Bicchierai, Irene, et al. "Using ontologies in the integration of structural, functional, and process perspectives in the development of safety critical systems." International Conference on Reliable Software Technologies. Springer, Berlin, Heidelberg, 2013.

23. COBIT, URL: http://www.isaca.org/cobit/pages/default.aspx; Accessed: February 2018.

24. The Business Rules Group. (2010). The Business Motivation Model, Business Governance in a Volatile World. Available at: http://www.businessrulesgroup.org/second_paper/BRG-BMM.pdf. Accessed: June, 2018.

25. ArchiMate (Version 2.4). Available at: http://archi.cetis.ac.uk/download.html, Accessed: April 2018. 
International Journal of Advances in Scientific Research and Engineering (ijasre), Vol 5 (4), April-2019

26. TOG (2012). ArchiMate 2.1 Specification. The Open Group. Available at: http://pubs.opengroup.org/architecture/archimate2-doc/chap10.html. Accessed: July 2018.

27. Quartel, Dick, et al. "A goal-oriented requirements modelling language for enterprise architecture." 2009 IEEE International Enterprise Distributed Object Computing Conference. IEEE, 2009.

28. Zachman, John A. "John Zachman's Concise Definition of The Zachman Framework (2008)." Zachman International (2014).

29. Proper, Henderik, and Marc M. Lankhorst. "Enterprise architecture-towards essential sensemaking." Enterprise Modelling and Information Systems Architectures (EMISAJ)9.1 (2014): 5-21.

30. Green, Nigel, and Carl Bate. Lost in translation: A handbook for information systems in the 21st century. New York: Evolved Technologist Press, 2007.

31. Coryn, Chris LS, et al. "A systematic review of theory-driven evaluation practice from 1990 to 2009." American journal of Evaluation 32.2 (2011): 199-226.

32. Huang, Jiewen, Daniel J. Abadi, and Kun Ren. "Scalable SPARQL querying of large RDF graphs." Proceedings of the VLDB Endowment 4.11 (2011): 1123-1134.

33. Gyongyi, Zoltan, Hector Garcia-Molina, and Jan Pedersen. Web content categorization using link information. Stanford, 2006.

34. McShane, Marjorie, and Sergei Nirenburg. "Use of ontology, lexicon and fact repository for reference resolution in Ontological Semantics." New Trends of Research in Ontologies and Lexical Resources. Springer, Berlin, Heidelberg, 2013. 157-185.

35. Lacher, Martin S., and Georg Groh. "Facilitating the Exchange of Explicit Knowledge through Ontology Mappings." FLAIRS conference. 2001.

36. Stumme, Gerd, and Alexander Maedche. "FCA-Merge: Bottom-up merging of ontologies." IJCAI. Vol. 1. 2001.

37. Keith, Mike, and Merrick Schincariol. "Advanced Queries." Pro JPA 2. Apress, Berkeley, CA, 2013. 307-336.

38. Gregor, Shirley, and Alan R. Hevner. "Positioning and presenting design science research for maximum impact." MIS quarterly (2013): 337-355.

39. Gregor, Shirley. "The nature of theory in information systems." MIS quarterly (2006): 611-642.

40. Venable, John R. "Design science research post hevner et al.: criteria, standards, guidelines, and expectations." International Conference on Design Science Research in Information Systems. Springer, Berlin, Heidelberg, 2010. 